\title{
Variable Length Space Time Coded Modulation
}

\author{
S. X. Ng, J. Wang, L.-L. Yang and L. Hanzo \\ School of ECS, University of Southampton, SO17 1BJ, UK. \\ Tel: +44-23-8059 3125, Fax: +44-23-8059 4508 \\ Email: \{sxn,jw02r,1ly,lh\}@ecs.soton.ac.uk, http://www-mobile.ecs.soton.ac.uk
}

\begin{abstract}
A Variable Length Space Time Coded Modulation (VL-STCM) scheme capable of simultaneously providing coding, multiplexing and diversity gains is proposed. The scheme advocated achieves its best performance for correlated sources, where the source symbols exhibit a nonuniform probability of occurrence. The source symbols are encoded using an optimal trellis encoder into variablelength modulated signals and mapped to both the spatial and time domains. More explicitly, the proposed VL-STCM arrangement is a jointly designed source coding, channel coding, modulation and spatial diversity/multiplexing scheme. It is shown that the higher the source correlation, the higher the achievable performance gain of the scheme. Furthermore, the performance of the VL-STCM scheme is about $6 \mathrm{~dB}$ better than that of the Fixed Length STCM (FL-STCM) benchmarker at a source symbol error ratio of $10^{-4}$.
\end{abstract}

\section{INTRODUCTION}

Shannon's separation theorem stated that source coding and channel coding is best carried out in isolation [1]. However, this theorem was formulated in the context of potentially infinitedelay, lossless entropy-coding and infinite block length channel coding. In practice, real-time wireless audio/video communications systems do not meet these ideal hypotheses. Explicitly, the source encoded symbols often remain correlated, despite the lossy source encoder's efforts to remove all redundancy. Furthermore, they exhibit unequal error sensitivity. In these circumstances, it is often more efficient to use jointly designed as well as optimised source and channel coders. Variable Length Codes (VLCs) [2] belong to the family of lowcomplexity source compression schemes, where the length of each VLC codeword is dependent on its probability of occurrence. VLCs are employed by most image coding standards [3, 4], although they are very sensitive to channel errors and to consequent loss of synchronisation due to their variable length nature. Even a single bit error is likely to induce a prolonged propagation errors and hence the data received after the bit error's position becomes error-infested. Furthermore, side information regarding the total number of VLC encoded

The financial support of the EPSRC, Swindon UK and the EU under the auspices of the PHOENIX as well as NEWCOM and NEXWAY projects is gratefully acknowledged. bits or symbols per transmission frame has to be conveyed to the receiver during each frame's transmission. Hence, a VLC scheme that does not suffer from error propagation and does not require the transmission of side information is desirable.

The wireless communication systems of future generations are required to provide reliable transmissions at high data rates in order to offer a variety of multimedia services. Space time coding schemes, which employ multiple transmitters and receivers, are among the most efficient techniques designed for providing high data rates by exploiting the high channel capacity potential of Multiple-Input Multiple-Output (MIMO) channels [5, 6]. More explicitly, Bell-lab's LAyered SpaceTime architecture (BLAST) [7] was designed for providing full transmitter-multiplexing gain, while Space-Time Trellis Codes (STTC) [8] were designed for providing full transmitterdiversity gain. Designing space-time coding schemes that are capable of providing both high data rates by achieving a high multiplexing gain, as well as reliable transmissions, with the advent of a diversity gain is highly desirable.

In this contribution, we proposed a jointly designed VLC and Space Time Coded Modulation (STCM) scheme, where two dimensional (2D) non-binary VLCs are proposed and transmitted by exploiting both the spatial and time domains. More specifically, the number of activated transmit antennas equals the number of non-binary symbols of the corresponding VLC codeword in the spatial domain, where each VLC codeword is transmitted during a single symbol period. Hence, the transmission frame length is determined by the fixed number of source symbols and therefore the proposed Variable Length STCM (VL-STCM) scheme does not exhibit synchronisation problems and does not require the transmission of side information. Additionally, the associated source correlation is converted into an increased product distance, hence resulting in an increased coding gain. Furthermore, the VL-STCM is capable of providing both multiplexing and diversity gains with the aid of multiple transmit antennas.

The rest of the paper is organised as follows. The overview of the space time coding technique advocated is given in Section 2, while the detailed description of the proposed VL-STCM scheme is presented in Section 3. In Section 4, the performance of VL-STCM is discussed and finally our conclusions are offered in Section 5. 


\section{SPACE TIME CODING OVERVIEW}

Let us consider a MIMO system employing $N_{t}$ transmit antennas and $N_{r}$ receive antennas. The signal to be transmitted from transmit antenna $m$, where $1 \leq m \leq N_{t}$, at discrete time index $t$ is denoted as $x_{m}[t]$. The received signal at receive antenna $n$, where $1 \leq n \leq N_{r}$, and at time instant $t$ can be modelled as:

$$
r_{n}[t]=\sqrt{E_{s}} \sum_{m=1}^{N_{t}} h_{n, m}[t] x_{m}[t]+w_{n}[t],
$$

where $E_{s}$ is the average energy of the signal constellation, $h_{n, m}[t]$ denotes the flat-fading channel coefficient between transmit antenna $m$ and receive antenna $n$ at time instant $t$, while $w_{n}[t]$ is the Additive White Gaussian Noise (AWGN) having zero mean and a variance of $N_{0} / 2$ per dimension. The amplitude of the modulation constellation points is scaled by a factor of $\sqrt{E_{s}}$, so that the average energy of the constellation points becomes unity and the expected Signal-to-Noise Ratio (SNR) per receive antenna is given by $\gamma=N_{t} E_{s} / N_{0}$ [9]. Let us denote the transmission frame length as $T$ symbol periods and define the spacetime encoded codeword as an $\left(N_{t} \times T\right)$ dimensional matrix $\mathbf{C}$ formed as:

$$
\mathbf{C}=\left[\begin{array}{cccc}
c_{1}[1] & c_{1}[2] & \ldots & c_{1}[T] \\
c_{2}[1] & c_{2}[2] & \ldots & c_{2}[T] \\
\vdots & \vdots & \ddots & \vdots \\
c_{N_{t}}[1] & c_{N_{t}}[2] & \ldots & c_{N_{t}}[T]
\end{array}\right]
$$

where the $t$ th column $\mathbf{c}[\mathbf{t}]=\left[c_{1}[t] c_{2}[t] \ldots c_{N_{t}}[t]\right]^{T}$ is the spacetime signal transmitted at time instant $t$ and the $m$ th row $\mathbf{c}_{\mathbf{m}}=\left[c_{m}[1] c_{m}[2] \ldots c_{m}[T]\right]$ is the signal transmitted from antenna $m$. The Pair-Wise Error Probability (PWEP) of erroneously detecting $\mathbf{E}$ instead of $\mathbf{C}$ is upper bounded at high SNRs by $[8,10]$ :

$$
p(\mathbf{C} \rightarrow \mathbf{E}) \leq \frac{1}{2}\left(\frac{E_{s}}{4 N_{0}}\right)^{-E_{H} \cdot N_{r}}\left(E_{P}\right)^{-N_{r}},
$$

where $E_{H}$ is referred to as the effective Hamming distance, which quantifies the transmit diversity order and $E_{P}$ is termed as the effective product distance, which quantifies the coding advantage of a spacetime code. It was shown in [11] that both the Hamming distance and the product distance of a block code can be preserved, when the block code is transmitted diagonally across the space-time grid. The mechanism of the diagonal transmission across the space-time grid will be exemplified in Section 3.1 in the context of Figures 1 and 2. Hence, a full transmitter-diversity STTC scheme can be realised, when the Hamming distance of the block code equals to the number of transmitters. Furthermore, the diagonal transmission of a block code also results in the minimum number of required trellis states [11], hence a full transmitter-diversity STTC scheme having the minimum decoding complexity is attained.

Let us now commence our detailed discourse on the proposed VL-STCM scheme in the following section.

\section{VARIABLE LENGTH SPACE TIME CODED MODULATION}

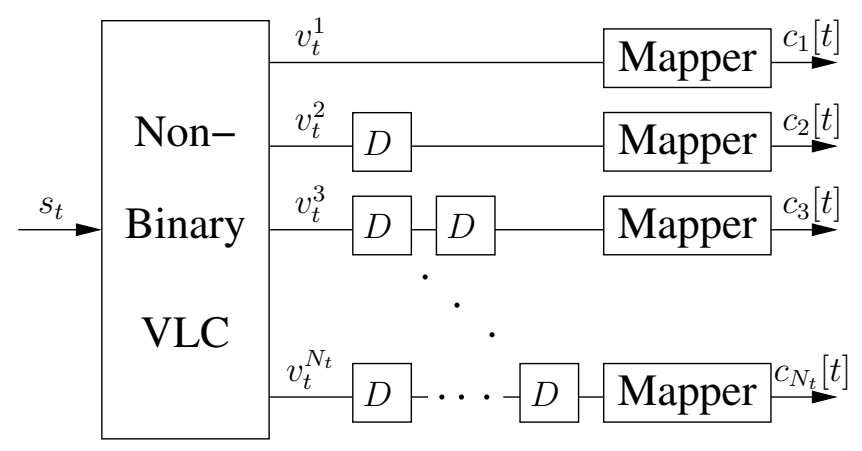

Figure 1: Block diagram of the VL-STCM encoder.

The block diagram of the VL-STCM scheme is illustrated in Figure 1. A non-binary VLC codeword $\mathbf{v}_{\mathbf{t}}=\left[v_{t}^{1} v_{t}^{2} \ldots v_{t}^{N_{t}}\right]^{T}$ is assigned to each of the source symbols $s_{t}$ generated by the source at time instant $t$, where $s_{t} \in\left\{1, \ldots, N_{s}\right\}, N_{s}$ denotes the number of possible source symbols and each component of the VLC codeword $\mathbf{v}_{\mathbf{t}}$ is represented by a non-binary rather than binary symbol. The non-binary VLC codeword $\mathbf{v}_{\mathbf{t}}$ is transmitted diagonally across the space-time grid with the aid of appropriate-length shift registers denoted as $D$ in Figure 1. More specifically, the number of shift registers used for transmit antenna $m$, where $m=\left\{1,2, \ldots, N_{t}\right\}$, is given by $m-1$. Hence, the proposed VL-STCM scheme transmits a non-binary two-dimensional (2D) VLC diagonally across the space-time grid in order to achieve a transmitter-diversity order which is identical to the Hamming distance of the 2D VLC plus a coding advantage quantified by the product distance of the 2D VLC as well as a multiplexing gain, when the number of possible source symbols $N_{s}$ is higher than the number of modulation levels $M$.

\subsection{Code Design}

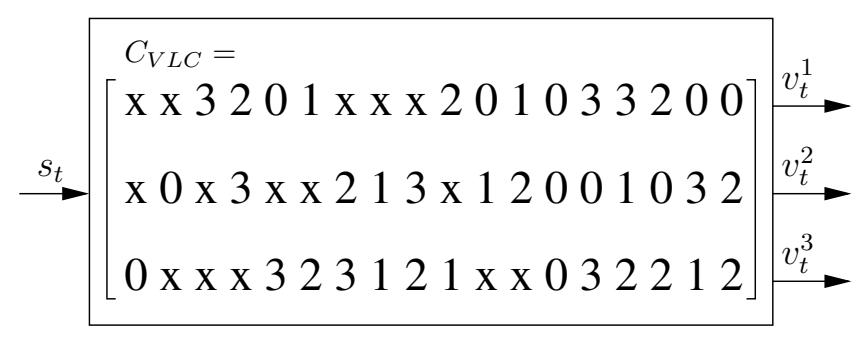

Figure 2: The $N_{s}=18$ possible VLC codewords $\mathbf{v}_{\mathbf{t}}$ of the codeword matrix in the non-binary $2 \mathrm{D}$ VLC $C_{V L C}$ employing 4PSK modulation, which signals the $N_{s}=18$ possible source symbols with the aid of using $N_{t}=3$ transmit antennas.

An example of the 2D non-binary VLC codeword matrix, $C_{V L C}$, is depicted in Figure 2 when there are $N_{s}=18$ possible source symbols and $N_{t}=3$ transmit antennas. More 
explicitly, each column of the $(3 \times 18)$-dimensional matrix $C_{V L C}$ corresponds to one codeword and the elements in the matrix represent the 4PSK symbols to be transmitted by the different antennas, while ' $x$ ' represents 'no transmission'. 'No transmission' implies that the corresponding transmitter antenna sends no signal. The VLC codeword $\mathbf{v}_{\mathbf{t}}$ corresponding to source symbol $s_{t}=j, j=1,2, \ldots, 18$, at time instant $t$ is given by the $j$ th column of the matrix $C_{V L C}$. Hence, each of the $N_{t}=3$ components of $\mathbf{v}_{\mathbf{t}}$ may assume one of the following values $v_{t}^{i} \in\{\mathrm{x}, 0,1,2,3\}$, where $i \in\left\{1,2, \ldots, N_{t}\right\}$ when 4 PSK modulation is employed ${ }^{1}$. Note that more transmission energy is saved, which may be reallocated to 'active' symbols, when there are more 'no transmission' symbols in a VLC codeword $\mathbf{v}_{\mathbf{t}}$. Therefore, the columns of the matrix $C_{V L C}$, which are the VLC codewords, and the source symbols are specifically arranged, so that the more frequently occurring source symbols are assigned to VLC codewords having more 'no transmission' components, in order to save transmit energy.

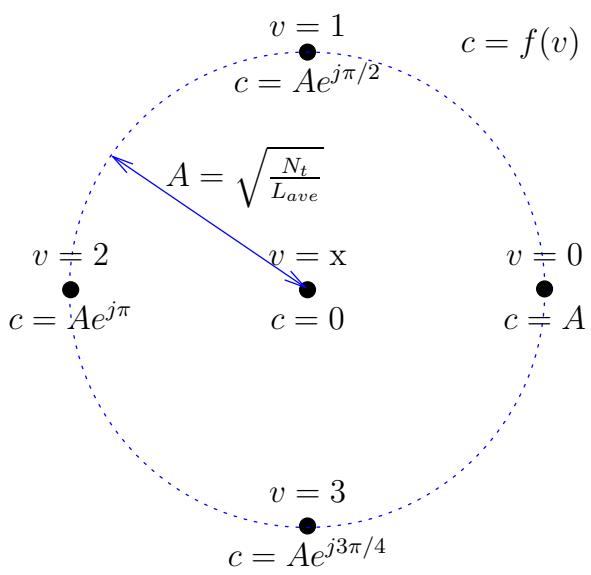

Figure 3: The signal mapper of the VL-STCM encoder of Figure 2.

The signal mapper blocks of the VL-STCM scheme of Figure 1 are characterised in Figure 3. Note that the "no transmission' symbol is actually represented by the origin of the Euclidean space, i.e. we have $c=f(v=\mathrm{x})=0$, where $f($.$) is$ the function mapping the 4PSK symbols to the $N_{t}$ number of transmit antennas. Furthermore, the amount of energy saving can be computed from:

$$
A=\sqrt{\frac{N_{t}}{L_{\text {ave }}}},
$$

where $L_{\text {ave }}$ is the average codeword length of the VLC codeword matrix $C_{V L C}$. As an example, we use the $N_{s}=18$ source symbol probabilities of $P(s)=\{0.1776,0.158,0.1548$, $0.1188,0.1162,0.077,0.0682,0.0388,0.0374,0.0182,0.017$, $0.0068,0.0066,0.002,0.0016,0.0007,0.0002,0.0001\}$. Hence,

\footnotetext{
${ }^{1}$ At this early phase of the research we set aside the related crest-factor problems for future work.
}

we have $L_{\text {ave }}=\sum_{s=1}^{N_{s}} P(s) L(s)=1.5208$ for the $C_{V L C}$ matrix of Figure 2, where $L(s)$ is the number of 'active' transmitted symbols in the VLC codeword assigned to source symbol $s$ according to Figure 2. Hence, in this specific example a power saving of $20 \log _{10}(A)=2.9505 \mathrm{~dB}$ can be obtained. Note however that the more correlated the source, the lower the average codeword length, and hence the higher the power saving. In order to normalise the average energy of the multiple transmitter-based modulation constellations to unity, we have to scale the 4PSK constellation of each transmitters such that the radius of the 4PSK constellation becomes $A=$ $\sqrt{3} 1.5208=1.4045$.

As we can see from Figures 1 and 2, each of the 18 nonbinary VLC codewords $\mathbf{v}_{\mathbf{t}}=\left[\begin{array}{lll}v_{t}^{1} & v_{t}^{2} & v_{t}^{3}\end{array}\right]^{T}$ is transmitted using $N_{t}=3$ transmitters, where the $m$ th element of each VLC codeword, for $1 \leq m \leq N_{t}$, is delayed by $(m-1)$ shift register cells, before it is transmitted through the $m$ th transmit antenna. Hence, the $N_{t}$ number of components of each VLC codeword are transmitted on a diagonal of the spacetime codeword matrix of Equation 2. Since the VLC codewords are transmitted diagonally, the transmitted signal at the $m$ th antenna, for $1 \leq m \leq N_{t}$, at a particular time-instant $t$ is given by $c_{m}[t]=f\left(v_{t-m+1}^{m}\right)$. Hence, for this specific case the spacetime codeword matrix of Equation 2 can be written as:

$\mathbf{C}=\left[\begin{array}{cccccc}\ldots & f\left(v_{t}^{1}\right) & f\left(v_{t+1}^{1}\right) & \ldots & f\left(v_{t+N_{t}-1}^{1}\right) & \ldots \\ \cdots & f\left(v_{t-1}^{2}\right) & f\left(v_{t}^{2}\right) & \ldots & f\left(v_{t+N_{t}-2}^{2}\right) & \ldots \\ \vdots & \vdots & \vdots & \ddots & \vdots & \vdots \\ \cdots & f\left(v_{t-N_{t}+1}^{N_{t}}\right) & f\left(v_{t-N_{t}+2}^{N_{t}}\right) & \ldots & f\left(v_{t}^{N_{t}}\right) & \ldots\end{array}\right]$

It can be shown based on [11] that the minimum Hamming distance $E_{H}$ of the VL-STCM scheme is given by:

$$
E_{H \min }=d_{H}^{V L C}
$$

where $d_{H}^{V L C}$ is the symbol-based minimum Hamming distance of the 2D VLC employed. The symbol-based Hamming distance between any two VLC codewords, which are selected from the columns of the codeword matrix $C_{V L C}$ of Figure 2, is given by the number of different symbols - rather than bits - between the two VLC codewords. For example, the symbolbased Hamming distance between the first and the last columns of $C_{V L C}$ shown in Figure 2 is three, where the "no transmission' symbol ' $\mathrm{x}$ ' is included as the fifth symbol. On the other hand, the minimum product distance $E_{P}$ of VL-STCM scheme can be computed as [11]:

$$
E_{P \min }=\min _{1 \leq s<\tilde{s} \leq N_{s}} \prod_{m \in \xi}\left|f\left(v^{m}\right)-f\left(\tilde{v}^{m}\right)\right|^{2}
$$

where $\xi$ is the set for transmit antenna index $m$ with $v^{m} \neq \tilde{v}^{m}$ for $1 \leq m \leq N_{t}$, while $\left[v^{1} \ldots v^{m}\right]^{T}$ and $\left[\tilde{v}^{1} \ldots \tilde{v}^{m}\right]^{T}$ are two VLC codewords associated with the source symbols $s$ and $\tilde{s}$, respectively. Hence the design of the 2D non-binary VLC code of Figure 2 is based on first designing a set of codeword matrices, which have the maximum achievable $E_{H \text { min }}$ and $E_{P \text { min }}$ values at a given $N_{s}$ and $N_{t}$ combination. Then we opt for the specific codeword matrix, which has the highest number 
of 'no transmission' symbols at the given $E_{H \text { min }}$ and $E_{P \text { min }}$ combination.

In the example of Figure 2 we have $E_{H \text { min }}=d_{H}^{V L C}=2$ and $E_{P \min }=3.89$, which were found to be the maximum achievable values at $N_{s}=18$ and $N_{t}=3$ by our exhaustive computer search for the best solution. Furthermore, the effective throughput of the VL-STCM scheme is given by $\log _{2}\left(N_{s}\right)=$ 4.17 bits per symbol (bps), which is significantly higher than $\log _{2}(4)=2 \mathrm{bps}$ in the case of classic 4PSK modulation. Therefore, firstly a transmit diversity order of $E_{H \text { min }}=2$ was attained. Secondly, a coding advantage directly related to the value of $E_{P \text { min }}=3.89$ was achieved. Thirdly, a multiplexing gain can also be attained for $N_{s}>M$, where $M$ is the number of modulation levels in the original modulation scheme. Additionally, the correlation of the source only affects the specific mapping of the different-probability source symbols to the 2D VLC codewords of Figure 2, but not the design of the 2D VLC itself. Therefore, the higher the source correlation, the lower the average codeword length $L_{\text {ave }}$. Hence, a higher power saving can be obtained, when the source is more correlated, since the amount of energy saving is given by Equation 4, where $N_{t}$ is fixed.

\subsection{Benchmarker}

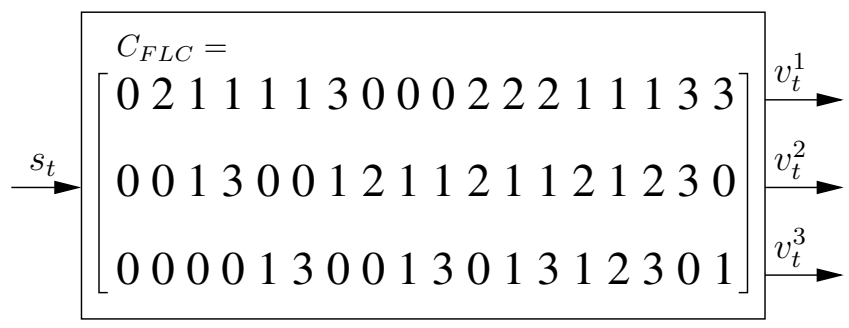

Figure 4: The codeword matrix of the non-binary 2D FLC $C_{F L C}$ employing 4PSK modulation, having $N_{s}=18$ number of possible source symbols and using $N_{t}=3$ transmit antennas.

In order to benchmark the proposed VL-STCM scheme of Figure 2, a Fixed-Length STCM (FL-STCM) benchmarker was created. The codeword matrix of the Fixed Length Code (FLC) is shown in Figure 4, where again, 4PSK modulation was employed in conjunction with $N_{s}=18$ and $N_{t}=3$. In the FLC all $N_{t}$ transmit antennas are active at all time instants, hence there are only $M=4$ phasors in the modulation constellation and no power saving is achieved due to the absence of 'no transmission' symbols. Similar to the VLC scheme of Figure 2, this was designed by exhaustive computer search, evaluating Equations 5 and 6 for all possible combinations of the 4PSK symbols.

At $N_{s}=18$, we have $E_{H \text { min }}=1$ and $E_{P \text { min }}=2$, which are the maximum achievable values for the FLC at a given combination of $N_{s}=18$ and $N_{t}=3$. However, these values are significantly smaller than the $E_{H \text { min }}$ and $E_{P \text { min }}$ of the 2D VLC. Nonetheless, both FL-STCM and VL-STCM achieve the same multiplexing gain due to having the same average throughput of $4.17 \mathrm{bps}$.

\subsection{Decoding}

At the receiver, both the Viterbi and the MAP algorithms may be used by the trellis-based decoder. The trellis states are defined by the contents of the shift register cells $D$ shown in Figure 1. Note that each shift register cell may hold five possible values, namely $\{\mathrm{x}, 0,1,2,3\}$ for the VL-STCM and four 4PSK values for the FL-STCM scheme. However, not all consecutive state sequences are possible due to the constraint imposed by the 2D VLC of Figure 2 or by the FLC code of Figure 4. More specifically, there are 90 and 44 possible trellis states in the trellis of the VL-STCM and FL-STCM schemes, respectively. Hence, the decoding complexity of the VL-STCM based on the $C_{V L C}$ matrix of Figure 2 is about twice that of the FL-STCM based on the $C_{F L C}$ matrix of Figure 4 . The log-domain symbol metrics can be computed based on the PDF of the noise as:

$$
\operatorname{Pr}(\mathbf{c}[t] \mid \mathbf{r}[t])=-\frac{1}{N_{0}} \sum_{n=1}^{N_{r}}\left|r_{n}[t]-\sqrt{E_{s}} \sum_{m=1}^{N_{t}} h_{n, m}[t] c_{m}[t]\right|^{2},
$$

where $\mathbf{r}[t]=\left[r_{1}[t] \ldots r_{N_{r}}[t]\right]$ is the received signal vector at time-instant $t, \mathbf{c}[t]=\left[c_{1}[t] \ldots c_{N_{t}}[t]\right]$ is the $N_{t}$-element space-time coded symbol and $c_{m}[t]=f\left(v_{t-m+1}^{m}\right)$. When the different source symbols' probability of occurrence $P(d)$ is different, it can be used as the a priori probability for assisting the trellis decoder. Hence, the log-domain trellis branch transition metrics of the transition from the previous state $S^{-1}$ to the current state $S$ may be computed as:

$$
\gamma\left(S^{-1}, S\right)=\operatorname{Pr}(\mathbf{c}[t] \mid \mathbf{r}[t])+\operatorname{Pr}(s),
$$

where $\mathbf{c}[t]$ and $s$ are the associated codeword and source symbol for that trellis branch, while $\operatorname{Pr}(\mathbf{c}[t] \mid \mathbf{r}[t])$ is given by Equation 7 and $\operatorname{Pr}(s)=\ln P(s)$ is the log-domain a priori probability of the source symbol $s$.

It is worth mentioning that it is possible to design a VLSTCM scheme, which has a higher minimum product distance, $E_{P \text { min }}$, at the cost of a higher decoding complexity. More explicitly, the design of the proposed VL-STCM is based on the search for an optimum non-binary $2 \mathrm{D}$ VLC matrix $C_{V L C}$, as shown in Figure 2, and its trellis is based on the shift-registers shown in Figure 1. By contrast, a VL-STCM scheme having a higher $E_{P \text { min }}$ can be designed based on the search of an optimum space-time codeword matrix $\mathbf{C}$, given by Equation 2, using a trellis that has a higher number of trellis states.

\section{SIMULATION RESULTS}

Let us now evaluate the performance of both the VL-STCM and FL-STCM schemes in terms of their source Symbol Error Ratio (SER) versus the Signal to Noise Ratio (SNR) per bit, which is given by $E_{b} / N_{0}=\gamma / \eta$, where $\gamma$ is the SNR 
per receive antenna and $\eta=\log _{2}(18)=4.17$ is the effective information throughput.

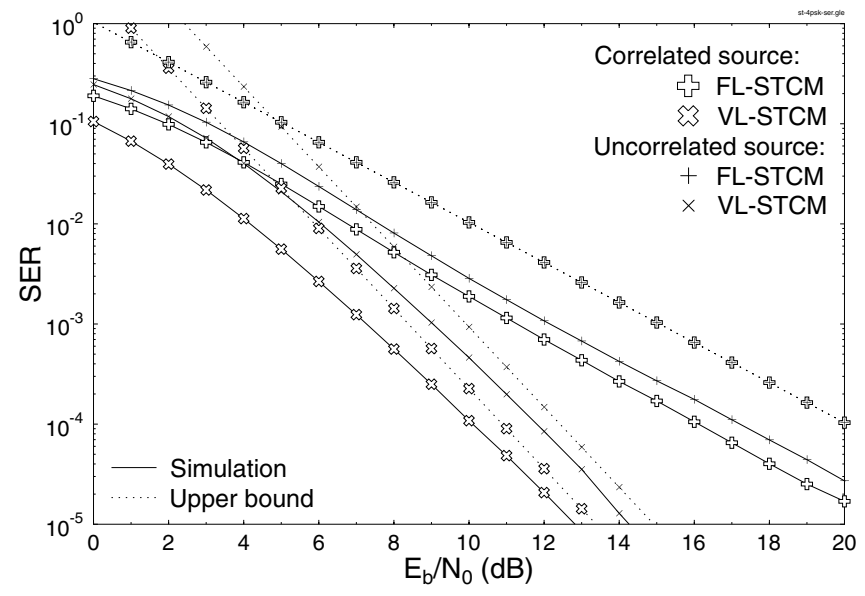

Figure 5: SER versus $E_{b} / N_{0}$ performance of the VL-STCM and FL-STCM schemes, when communicating over uncorrelated Rayleigh fading channels using 4PSK, $N_{t}=3$ and $N_{r}=2$.

Figure 5 depicts the SER versus $E_{b} / N_{0}$ performance of the VL-STCM and FL-STCM schemes, when communicating over uncorrelated Rayleigh fading channels using 4PSK, three transmitters and two receivers. Note that when the source is uncorrelated, we have $L_{\text {ave }}=2.1667$ and $A_{0}=\sqrt{\frac{N_{t}}{L_{\text {ave }}}}=$ 1.38 for the VL-STCM scheme, hence the power saving of VL-STCM is given by $20 \log _{10}\left(A_{0}\right)=1.4133 \mathrm{~dB}$ and the effective product distance is given by $E_{P \text { min }}=1.92$. By contrast, the results for the correlated source investigated are based on the source symbol probabilities $P(s)$ defined in Section 3. The upper bounds of the PWEP defined in Equation 3 are also shown in Figure 5 for comparison with the simulation results. As we can see from Figure 5, the performance of VLSTCM is about $1.7 \mathrm{~dB}$ better, when the source is correlated as compared to when the source is uncorrelated. This is due to the higher achievable $E_{P \text { min }}$ value, when the source is correlated. Observe in Figure 5 that the PWEP upper bounds of the VL-STCM are close to the simulation results. By contrast, the performance of the FL-STCM is about $1.1 \mathrm{~dB}$ better, when the source is correlated compared to the uncorrelated source. This is because the branch transition metrics in Equation 8 benefit from the different source symbol probabilities, when the source is correlated.

By comparing the simulation performance of VL-STCM and FL-STCM at SER $=10^{-4}$, the performance of VL-STCM is approximately $6 \mathrm{~dB}$ and $5.4 \mathrm{~dB}$ better than that of FL-STCM, when employing correlated and uncorrelated sources, respectively. Again, this coding gain is achieved at the cost of approximately doubling the decoding complexity from 44 to 90 trellis states.

\section{CONCLUSIONS}

A variable length space time coded modulation design was proposed, where a jointly designed optimal trellis codec was employed, instead of using a separate suboptimal source codec and channel codec pair. The joint design of source-coding and space-time coded modulation was shown to achieve both spatial diversity and multiplexing gain, as well as coding gain at the same time. The variable length structure of the individual codewords mapped to the maximum $N_{t}$ transmit antennas imposes no synchronisation and error propagation problems. Furthermore, it provides an efficient design that is capable of increasing the effective product distance of the scheme, especially when the source is correlated. An overall performance gain of $6 \mathrm{~dB}$ was attained by the VL-STCM scheme over the FL-STCM benchmarker scheme at $\mathrm{SER}=10^{-4}$. The proposed VL-STCM design may be employed for higher-order modulation schemes by first designing a two-dimensional VLC mapping 16-ary or even high-order modulation constellations to the $N_{t}$ transmit antennas. The proposed VL-STCM may also be employed as a constituent code in turbo equalisation systems or in parallel concatenated turbo coding architectures for the sake of attaining further iteration gains.

\section{REFERENCES}

[1] C. Shannon, Mathematical Theory of Communication. University of Illinois Press, 1963.

[2] L. Hanzo, P.J. Cherriman and J. Street, Wireless Video Communications: Second to Third Generation Systems and Beyond. NJ, USA : IEEE Press., 2001.

[3] ITU-T, Recommendation H.263: Video Coding for Low Bitrate Communication, March 1996.

[4] International Standards Organization, ISO/IEC CD 13818 MPEG 2 International Standard, Information Technology, Generic Coding of Moving Video and Associated Audio Information, Parts 1-3.

[5] G. Foschini Jr. and M. Gans, "On limits of wireless communication in a fading environment when using multiple antennas," Wireless Personal Communications, vol. 6, pp. 311-335, March 1998.

[6] E. Telatar, "Capacity of multi-antenna Gaussian channels," European Transactions on Telecommunication, vol. 10, pp. 585-595, Nov-Dec 1999.

[7] G. J. Foschini, Jr., "Layered Space-time architecture for wireless communication in a fading environment when using multi-element antennas," Bell Labs Technical Journal, pp. 41-59, 1996.

[8] V. Tarokh, N. Seshadri and A. R. Calderbank, "Space-time Codes for High Rate Wireless Communication: Performance analysis and code construction," IEEE Transactions on Information Theory, vol. 44, pp. 744-765, March 1998.

[9] A. F. Naguib, V. Tarokh, N. Seshadri and A. R. Calderbank, "A SpaceTime Coding Modem for High-Data-Rate Wireless Communications," IEEE Journal on Selected Areas in Communications, vol. 16, pp. 1459 1478, October 1998.

[10] D. Divsalar and M.K. Simon, "Trellis coded modulation for 4800-9600 bits/s transmission over a fading mobile satellite channel," IEEE Journal on Selected Areas in Communications, vol. 5, pp. 162-175, February 1987.

[11] M. Tao and R. S. Cheng, "Diagonal Block Space-time Code Design for Diversity and Coding Advantage over Flat Rayleigh Fading Channels," IEEE Transactions on Signal Processing, pp. 1012-1020, April 2004. 